\title{
Sphenoid sinus organized hematoma with cranial neuropathies masquerading as a malignancy: A case report
}

\author{
YU-HSUAN LIN ${ }^{1}$, PO-CHIN WANG ${ }^{2}$ and YAOH-SHIANG LIN ${ }^{1,3}$ \\ Departments of ${ }^{1}$ Otolaryngology - Head and Neck Surgery, and ${ }^{2}$ Radiology, Kaohsiung Veterans General Hospital, \\ Kaohsiung $813 ;{ }^{3}$ Department of Otolaryngology - Head and Neck Surgery, \\ National Defense Medical Center, Taipei 114, Taiwan, R.O.C.
}

Received November 19, 2014; Accepted October 5, 2015

DOI: $10.3892 / \mathrm{ol} .2016 .4475$

\begin{abstract}
Sinonasal organized hematoma (SNOH) is rarely encountered in clinical practice. The disease demonstrates a high tendency for occurrence in East Asian individuals, and in the majority of cases, is located in the maxillary sinus. The current report presents the case of an 81-year-old female who developed a space-occupying lesion, which masqueraded as a skull base malignancy, following surgery for the treatment of isolated sphenoid sinus aspergilloma. Subsequent endoscopic endonasal surgery confirmed the diagnosis of an $\mathrm{OH}$ of the sphenoid sinus. The patient recovered from all neurological deficits within two months, with the exception of the loss of visual perception. Although SNOH presents a diagnostic challenge, when physicians possess knowledge of its typical imaging features, this facilitates the achievement of a correct diagnosis and the prescription of optimal treatment.
\end{abstract}

\section{Introduction}

Sinonasal organized hematoma (SNOH) was initially described in 1917 by Tadokoro (1) as a benign lesion characterized by an expansile nature and locally aggressive behavior, capable of mimicking a potentially dangerous neoplastic process (2-5). The diagnosis of $\mathrm{SNOH}$ is challenging, even given the integration of multiple informative symptoms (2). Computed tomography (CT) and magnetic resonance

Correspondence to: Professor Yaoh-Shiang Lin, Department of Otolaryngology - Head and Neck Surgery, Kaohsiung Veterans General Hospital, 386 Ta-Chung First Road, Kaohsiung 813, Taiwan, R.O.C.

E-mail:mdlinys@gmail.com

Abbreviations: SNOH, sinonasal organized hematoma; $\mathrm{OH}$, organized hematoma; CT, computed tomography; MRI, magnetic resonance imaging; T1WI, T1-weighted imaging; T2WI, T2-weighted imaging; ISSA, isolated sphenoid sinus aspergilloma

Key words: sinonasal organized hematoma, sphenoid sinus, computed tomography, magnetic resonance imaging, endoscopic endonasal surgery imaging (MRI) are useful for the assessment of potential cases of $\mathrm{SNOH}(2,5)$, and usually reveal typical bony changes and a distinct speckled pattern that exhibits various signal intensities, respectively. The hallmarks of $\mathrm{SNOH}$ are typically a dark peripheral rim on T2-weighted imaging (T2WI), and a nodular and patchy enhancement in post-contrast T1-weighted imaging (T1WI) $(2,5)$. The successful treatment of $\mathrm{SNOH}$ relies on complete surgical excision $(3,5)$. However, one notable and unexplained anomaly is that the disease demonstrates a high tendency for occurrence in the maxillary sinus $(3,5-7)$. Only one case involving the sphenoid sinus, an intricately structured space in close vicinity to vital structures, has been reported to date (4). To the best of our knowledge, the current report presents the first case of an $\mathrm{OH}$ of the sphenoid sinus with multiple cranial nerve involvement, which was identified following surgery for the treatment of isolated sphenoid sinus aspergillosis (ISSA). The disease was successfully treated using endoscopic endonasal surgery, yielding a positive outcome for the patient.

\section{Case report}

An 81-year-old woman presented as an inpatient to the Kaohsiung Veterans General Hospital (Kaohsiung, Taiwan) in October 2013, with a 2-year history of an excruciating headache, which was described as deep-seated and throbbing. The patient had subsequently developed progressive deterioration of visual acuity in the right eye and drooping of the right eyelid, although the total duration of these symptoms was uncertain. The medical history of the patient noted the use of endoscopic sinus surgery for the treatment of ISSA 3 years previously, and a long-standing history of chronic kidney disease. Upon examination, besides optic atrophy, the patient's right eye exhibited complete ptosis and mydriasis with a sluggish pupillary reaction. The eye was positioned looking down and out, and the orbit could not be adducted. These symptoms were compatible with a diagnosis of compressive occulomotor nerve palsy. The nasal endoscopy revealed a friable mass with superficial telangiectasia straddling the torus tubarius and extending forward (Fig. 1). Non-contrast CT revealed an aggressively enlarging lesion in the midline skull base, with destructive bony structures (Fig. 2). Distinctive internal heterogeneous hyperintensity 


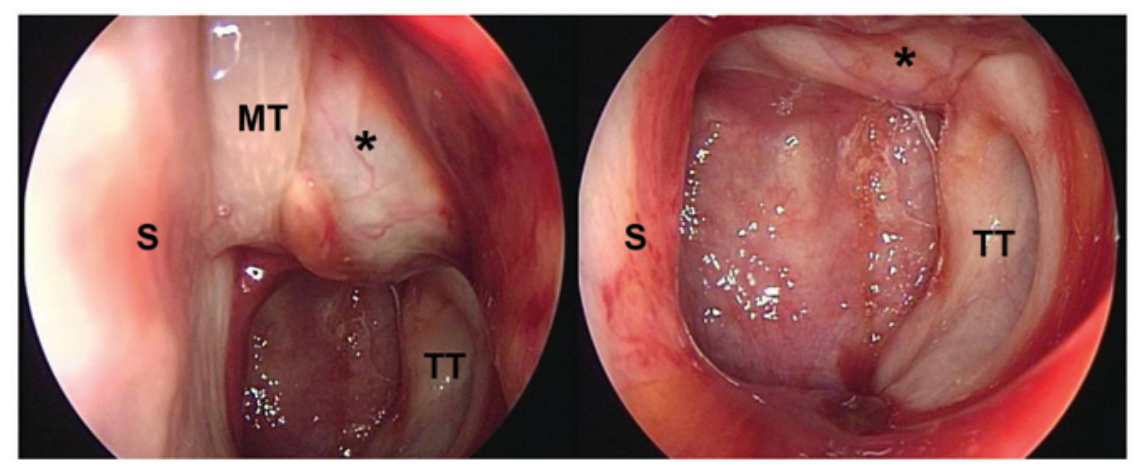

Figure 1. Sinoscopic examination at patient presentation revealing a friable lesion $(*)$ between the MT and the lateral wall of the nasal cavity, and straddling the TT. S, septum; MT, middle turbinate; TT, torus tubarius.
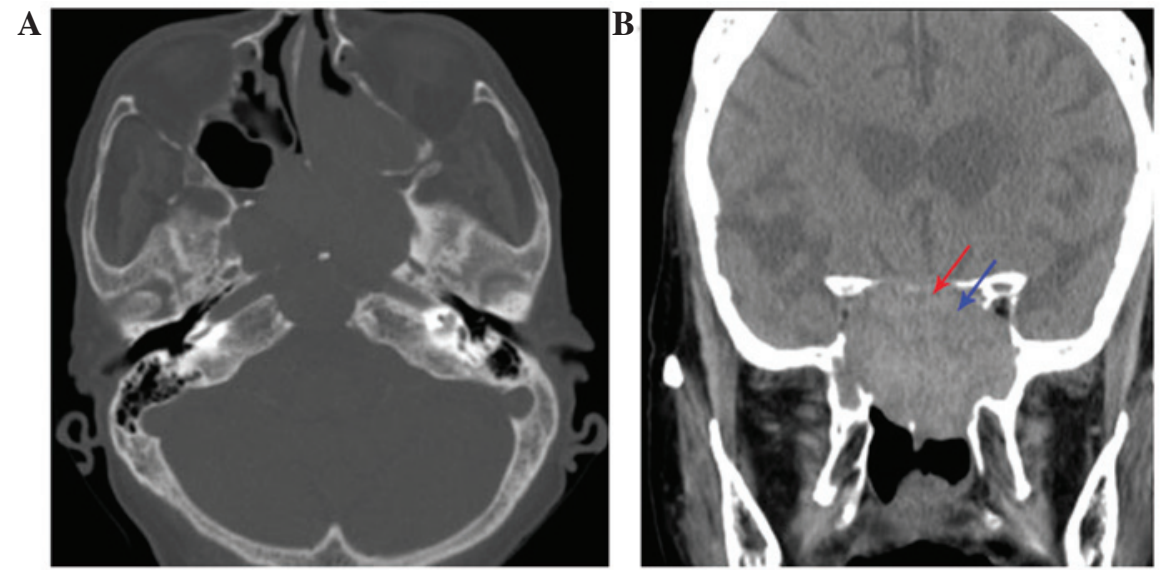

Figure 2. CT images. (A) Bony algorithm CT image revealing an expansile neoplasm of the sphenoid sinus, extending forward to the left ethmoid sinus, with erosion of adjacent skeletal structures. (B) Coronal non-contrast CT image demonstrating partial fat tissue effacement of the right superior orbital fissure and total obliteration of the right optic canal, compatible with clinical optic and oculomotor compressive neuropathies. Red arrow, left optic canal. Blue arrow, left superior orbital fissure. CT, computed tomography.

and a hypointense peripheral rim on T2WI, and a patchy enhancement pattern on post-contrast T1W1 were observed (Fig. 3). Routine laboratory data revealed normal coagulation and pituitary function profiles, including human growth hormone $(0.23 \mathrm{ng} / \mathrm{ml}$; normal range, $0.0-16 \mathrm{ng} / \mathrm{ml})$, luteinizing hormone $(31.35 \mathrm{mIU} / \mathrm{ml}$; normal range, $11.3-38.7 \mathrm{mIU} /$ $\mathrm{ml})$, follicle stimulating hormone $(74.09 \mathrm{mIU} / \mathrm{ml}$; normal range, $31.8-134 \mathrm{mIU} / \mathrm{ml})$ and free thyroxine $(1.58 \mathrm{ng} / \mathrm{dl}$; normal range, $0.8-1.9 \mathrm{ng} / \mathrm{dl})$. Only high sensitivity-thyroid-stimulating hormone levels were abnormal $(0.245 \mathrm{uIU} / \mathrm{ml}$; normal range, $0.4-4.0 \mathrm{uIU} / \mathrm{ml}$ ).

In order to optimize surgical planning, the patient underwent a biopsy, which yielded a negative result. The patient subsequently underwent endoscopic endonasal surgery to resect the lesion; a posterior ethmoidectomy, sphenoidotomy and septoplasty were required in order to gain complete surgical access. A well-capsulated, russet-colored and blood clot-containing tumor was removed in a piece-by-piece manner, leaving the periosteal layer of dura mater that protected the intracranial structures intact. The thinned bony components located above the right orbital apex and right optic canal were gently flaked off. The surgical extent was explored inferiorly to the upper clivus. A sample of the lesion sent for pathological analysis revealed an $\mathrm{OH}$ without any evidence of fungal hyphae (Fig. 4). The immediate post-operative course was uncomplicated, with resolution of the patient's headache. At the 2-month follow-up, the subject had recovered fully, with the exception of the lost vision.

Written informed consent was obtained from the patient for publication of the present case report and any accompanying images.

\section{Discussion}

Sphenoid disease is primarily a result of the extension of disease anteriorly from the ethmoid complex or posteriorly from the adjacent anatomical sites, including the sella turcica and the surrounding skull base structures (8). Isolated sphenoid sinus disease is rarely encountered and the associated symptoms are generally non-specific (8). Sphenoid disease frequently leads to nerve involvement, and the effects of masses causing compression, destruction or invasion lead to varying levels of impact on the extent and degree of neurological deficits (8). Etiologically, non-neoplastic lesions constitute the majority of cases, while fungal processes are the third most common cause (8). ISSA is a type of fungal sinus infection that is distinct from other categories of fungal rhinosinusitis, and is characterized by higher levels of occurrence in older women (9). A sphenoid sinusotomy is regarded to be the primary therapy $(8,9)$. To the best of our knowledge, the occurrence of an $\mathrm{OH}$ of the 

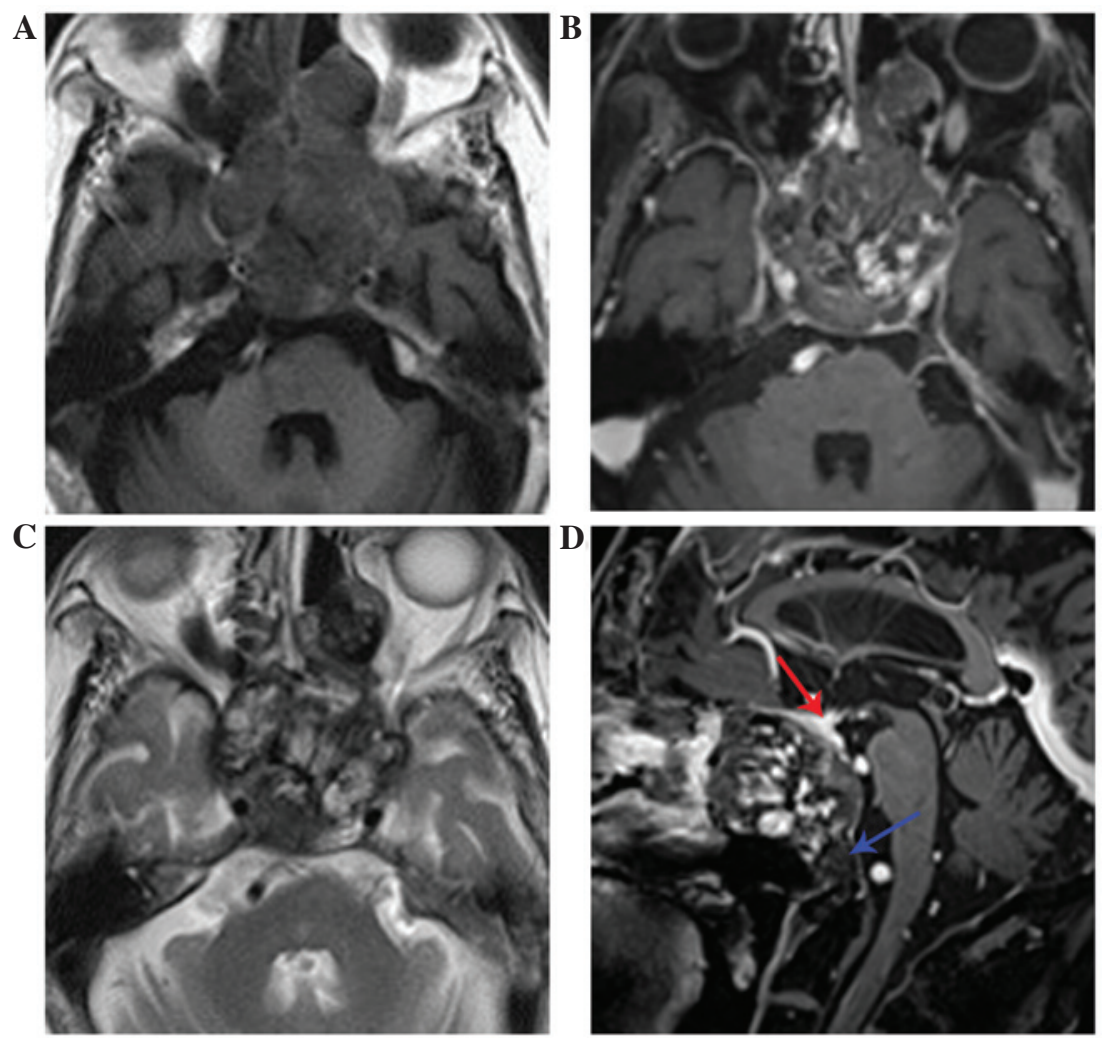

Figure 3. MRI images. (A) Simultaneous axial T1WI revealing heterogeneously mixed signals within the lesion, and typical patchy and (B) nodular enhancement following administration of gadolinium. (C) A dark peripheral rim with distinct internal mixture of varying signals on T2WI. (D) Contrast-enhanced sagittal T1WI revealing the extensive nature of the sinonasal organized hematoma, with the expansile tumor pushing the pituitary gland (red arrow) upwards, and concomitant caudal involvement of the clivus (blue arrow). T1WI, T1-weighted images; T2WI, T2-weighted images.

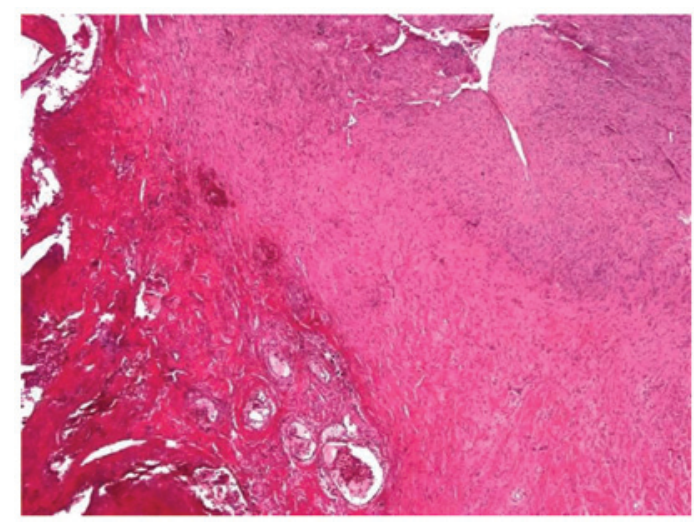

Figure 4. Pathological photomicrograph revealing a lesion consisting of numerous red blood cells mixed with neovascularization and granulation tissue. Hematoxylin and eosin stain; magnification, $\mathrm{x} 40$.

sphenoid sinus following ISSA surgery has not been previously reported $(2-6,8,9)$.

$\mathrm{SNOH}$ is rarely encountered in clinical practice (2-6). When it does occur, it is usually locally invasive and demonstrates a high tendency to occur in the maxillary sinus $(3,5,6,10)$. The incidence of $\mathrm{SNOH}$ appears to be most frequent in the population of East Asia $(3,5,6,10)$. The exact underlying etiological mechanisms remain to be elucidated $(4,5,6)$. Ozaki et al (11) advanced what is now the most widely-accepted hypothesis, the negative spiral theory $(3,6)$. The negative spiral theory assumes that an $\mathrm{OH}$ results from persistent negative intraluminal pressure following an initial episodic hemorrhage into a semi-closed cavity, leading to repeated rupturing of fragile mucosal vessels. Subsequent formation of a superficial fibrotic capsule prevents further reabsorption of the hematoma. Under the succession of biological healing processes, an $\mathrm{OH}$ emerges $(3,6)$. The progressive expansion of the $\mathrm{OH}$ results in augmentation of the sinus antrum, and therefore results in demineralization of the adjacent skeletal structures $(3,5)$.

Macroscopically, $\mathrm{SNOH}$ is described as a slow-growing, well-circumscribed and friable brownish mass. The histopathological findings reveal a mixture of neovascularization, fibrosis, hemorrhaging and hemosiderin deposition (5). A number of studies have attempted to identify an association between $\mathrm{SNOH}$ and sinonasal angiomatous polyps, a rare subtype of inflammatory sinonasal polyp, using radiology and histopathology $(10,12)$. However, this association remains to be fully elucidated. Returning to the present case, the causative etiology may be attributed to an obstructed sinus cavity with equivalent volume to the maxillary sinus, and repetitive post-operative hemorrhaging due to an inflammatory vascular injury. To the best of our knowledge, such a case was previously unknown, and has not been reported in the relevant literature (5). It may be postulated that the incidence of post-operative $\mathrm{SNOH}$ is underestimated. Long-term follow-up for patients undergoing endoscopic sinus surgery is necessary to provide an answer to this hypothesis.

CT possesses an advantage over MRI in terms of its ability to analyze the integrity of bone in detail (5). The density in unenhanced $\mathrm{CT}$ scans of $\mathrm{OH}$ is frequently hyperattenuated 
compared with masticator muscles. The hallmarks of $\mathrm{OH}$ are mucoperiosteal thickening, occasional calcification, convex bowing of natural skeletal architecture, cortical thinning or direct extension to adjacent structures sparing frank osseous destruction $(5,6)$. However, CT alone does not provide enough information to allow for the differentiation of $\mathrm{OH}$ from locally aggressive neoplasms. MRI exhibits marked superiority over $\mathrm{CT}$ in terms of its ability to determine the margin and true extent of tumor expansion. In addition, MRI possesses an advantage with regard to distinguishing adjacent secondary inflammation and nasal secretions from a tumor mass, and has the ability to display the corresponding pathological components within the lesion $(2,5)$. Typically, SNOH exhibits a mosaic of varying signal intensities in T1WI and T2WI, and heterogeneous enhancement in a patchy pattern following contrast administration $(2,5,10)$. For $\mathrm{SNOH}$, the most conclusive diagnostic finding is a hypointense zone surrounding the lesion on T2WI, indicating the pathological feature of a fibrous capsule $(2,5,10)$. Therefore, obtaining knowledge of the distinctive characteristic observations acquired using CT and MRI $(2,5,10)$ offers clinicians valuable anatomical and diagnostic informative clues that may allow for the achievement of a correct diagnosis of $\mathrm{SNOH}$.

To the best of our knowledge, the present study reports the first case of an $\mathrm{OH}$ of the sphenoid sinus in a patient who previously underwent ISSA surgery. The case highlights the requirement for physicians to include $\mathrm{SNOH}$ in the differential diagnosis for expansile sphenoid sinus disease, regardless of its low prevalence. Advances in techniques mean that the performance of endoscopic endonasal skull base surgery is clinically feasible and reliable for the treatment of patients exhibiting SNOH (7), particularly for cases in which patients exhibit compressive neuropathy, and timely intervention is required in order to avoid permanent sequelae (13). The present case additionally suggests that a preexisting inflammatory process and performance of previous surgery may have a role in multifaceted SNOH pathogenesis. However, increased experience with regard to the diagnosis and treatment of sphenoid $\mathrm{SNOH}$ is required in order to fully elucidate the underlying mechanisms of the disease.

In conclusion, although the pathogenesis and epidemiology of SNOH are not fully understood, the application of CT and MRI, combined with the involvement of a diagnosing physician who possesses familiarity with the classical imaging findings, allows for an accurate pre-operative diagnosis of $\mathrm{SNOH}$. Care should be exercised when interpreting the abnormal imaging results acquired from a sinus that has been operated on, in order to ensure that $\mathrm{SNOH}$ is not misidentified as recurrence or residual disease. Once a diagnosis has been reached, following consultation of the CT and MRI scans, the current understanding is that a complete surgical excision is the optimal treatment for $\mathrm{SNOH}$, yielding positive patient outcomes and rarely resulting in recurrence later in life.

\section{References}

1. Tadokoro K: Jogakudo ketsuryu ni tsuite. Dainichijibi 23: 359-360, 1917 (In Japanese).

2. Wu AW, Ting JY, Borgie RC, Busaba NY, Sadow PM, Juliano AF, Gray ST and Holbrook EH: Diagnostic characteristics of sinonasal organizing hematomas: Avoiding misdiagnosis. Int Forum Allergy Rhinol 3: 598-602, 2013.

3. Omura G, Watanabe K, Fujishiro Y, Ebihara Y, Nakao K and Asakage T: Organized hematoma in the paranasal sinus and nasal cavity - imaging diagnosis and pathological findings. Auris Nasus Larynx 37: 173-177, 2010

4. Nakagawa T, Kawai Y, Sakamoto T and Ito J: Organised haematoma of the sphenoid sinus mimicking a pituitary tumour. J Laryngol Otol 124: 83-85, 2010.

5. Kim EY, Kim HJ, Chung SK, Dhong HJ, Kim HY, Yim YJ, Kim ST, Jeon P and Ko YH: Sinonasal organized hematoma: $\mathrm{CT}$ and MR imaging findings. AJNR Am J Neuroradiol 29: 1204-1208, 2008.

6. Lee PK, Wu JK and Ludemann JP: Hemorrhagic pseudotumour of the maxillary sinus. J Otolaryngol 33: 206-208, 2004.

7. Lee LA, Huang CC and Lee TJ: Prolonged visual disturbance secondary to isolated sphenoid sinus disease. Laryngoscope 114: 986-990, 2004.

8. Ng YH and Sethi DS: Isolated sphenoid sinus disease: Differential diagnosis and management. Curr Opin Otolaryngol Head Neck Surg 19: 16-20, 2011.

9. Chakrabarti A, Denning DW, Ferguson BJ, Ponikau J, Buzina W, Kita H, Marple B, Panda N, Vlaminck S, Kauffmann-Lacroix C, et al: Fungal rhinosinusitis: A categorization and definitional schema addressing current controversies. Laryngoscope 119: 1809-1818, 2009.

10. Wang YZ, Yang BT, Wang ZC, Song L and Xian JF: MR evaluation of sinonasal angiomatous polyp. AJNR Am J Neuroradiol 33: 767-772, 2012.

11. Ozaki M, Sakai S and Ikeda H: Hemangioma of the nasal cavity and sinuses - a report of twenty five cases. Otolaryngol Head Neck Surg (Tokyo) 49: 53-58, 1977.

12. Yfantis HG, Drachenberg CB, Gray W and Papadimitriou JC: Angiectatic nasal polyps that clinically simulate a malignant process: report of 2 cases and review of the literature. Arch Pathol Lab Med 124: 406-410, 2000.

13. Castelnuovo P, Dallan I, Battaglia P and Bignami M: Endoscopic endonasal skull base surgery: Past, present and future. Eur Arch Otorhinolaryngol 267: 649-663, 2010. 